\title{
Diagnostic accuracy of the trans-abdominal ultrasound in the assessment of dysfunctional hemidiaphragm due to non- pulmonic etiology
}

\author{
Ahmed A. Baz ${ }^{1 *}$, Amal Abdel Hakam Mohammed Aglan ${ }^{1}$, Sabah Ahmed Mohammed ${ }^{2}$ and Youssriah Yahia Sabri ${ }^{1}$
}

\begin{abstract}
Background: To evaluate the role of the trans-abdominal ultrasound (TAUS) in the assessment of hemidiaphragmatic dysfunction — due to non-pulmonic causes—as compared to the conventional CT; 36 patients (22 males and 14 females; age range 5 to 84 years) were included in this study.

Results: In CT examination, the dysfunctional hemidiaphragm was considered when either a hernia/post-traumatic defects (present in 19.4\% of patients, of which 5.5\% were depicted on the right side and $13.8 \%$ were existing on the left side), or elevated copulae (present in $80.6 \%$ of patients, of which $58.8 \%$ were found on the right side (21 patients) and $22.2 \%$ were seen on the left side (8 patients).

The TAUS showed a high sensitivity, specificity, PPV, NPV, and over all accuracy $\{100 \%$ (95\% Cl=59.04 to 100.00\%), $100 \%(95 \% \mathrm{Cl}=88.06$ to $100.00 \%), 100 \%, 100 \%, 100 \%$ (95\% Cl $=90.26$ to $100.00 \%)$, respectively\} in detection of defects and hernias as compared to CT. A high sensitivity, specificity, PPV, NPV, and over all accuracy $\{96.55 \%$ (95\% $\mathrm{Cl}=82.24$ to $99.9 \%), 100 \%(95 \% \mathrm{Cl}=59.04$ to $100.00 \%), 100 \%, 87.5 \%(95 \% \mathrm{Cl}=50.50$ to $97.96 \%), 97.22 \%(95 \% \mathrm{Cl}=$ 85.47 to $99.93 \%)$, respectively\} were found in detection of thickness and motion abnormalities, and in the detection of subphrenic collections in cases with relevant elevated copula in CT.

Conclusion: Trans-abdominal ultrasound (TAUS) could accurately assess the diaphragmatic thickness and provides a real-time image of a dysfunctional hemidiaphragm due to a diaphragmatic and infradiaphragmatic causes as well as the diaphragmatic defects with high sensitivity and specificity in comparison to CT.
\end{abstract}

Keywords: Trans-abdominal, Ultrasound, CT, Diaphragm, Dysfunction

\section{Background}

The diaphragm is by far considered as the most important respiratory muscle constituting about $70 \%$ of the tidal inspiratory volume and is the sole skeletal muscle that has a continuous action with rhythmic nature being controlled by the respiratory control center in the brain stem, it also has additional non-respiratory functions that need an increased abdominal pressure $[1,2]$.

\footnotetext{
* Correspondence: ahmedbaz2012@yahoo.com

${ }^{1}$ Radiology Department, Faculty of Medicine, Cairo University, Cairo, Egypt Full list of author information is available at the end of the article
}

Diaphragmatic dysfunction usually involves one side and to a lesser extent, a bilateral dysfunction can occur. Nevertheless, hemidiaphragmatic impairment can be asymptomatic and is usually detected incidentally by either clinical examination or by non-targeted imaging studies; however, some patients may experience orthopnea or dyspnea on effort. On the other hand, a bilateral diaphragmatic affection (the less common type) is often symptomatic and is associated with ventilation problems [3].

Many diagnostic imaging modalities can be implemented in the assessment of the diaphragm; including plain radiography, CT, and MRI as anatomical imaging 
tools; however, X-ray fluoroscopy and MRI fluoroscopy can serve as functional imaging studies [4].

Trans-abdominal ultrasound (TAUS) had been included as an anatomical and functional imaging tool at the same time, as it provides an assessment of the diaphragmatic morphology in addition to the functional assessment of the diaphragmatic mobility [4].

Diaphragmatic disorders usually present radiologically as elevation, regardless of the cause; the diagnosis is based on static and dynamic imaging tools including the plain chest radiography, fluoroscopy, and TAUS $[4,5]$.

TAUS had provided a safe, non-invasive, and cheap imaging workup for anatomical and functional assessment of the diaphragm with accurate and reproducible results; moreover, it had no exposure to ionizing radiation like that in X-ray and CT fluoroscopy [6].

Diaphragmatic elevation has many causes that could be divided as supradiaphragmatic, diaphragmatic, and infradiaphragmatic; however, the TAUS had been described to have a limited role in the assessment of the supradiaphragmatic causes apart from some basal pleuro-pulmonary disorders, and eventually it is not traditionally used to assess the disease extent in such disorders [7].

In the present study, we were concerned with the accuracy measures of the TAUS evaluation of the unilateral diaphragmatic dysfunction as reported by CT due to diaphragmatic and infradiaphragmatic causes excluding the pulmonic (supradiaphragmatic) causes, as the TAUS had a limited role in this regard and might not even display the full extent of the detected disorders.

\section{Aim of the work}

The purpose of this study was to test the diagnostic accuracy of the TAUS in the evaluation of hemidiaphragmatic dysfunction-due to non-pulmonic causes-in comparison to the conventional CT.

\section{Methods}

The study was conducted on 36 patients who undergone CT chest examination and revealed a unilateral diaphragmatic affection (22 males and 14 females) from August 2018 till July 2019. The patients were referred to our department from the chest diseases department, outpatient clinics of chest, surgery and pediatric surgery outpatient clinic and emergency unit after their clinical assessment for ultrasound evaluation of their affected hemidiaphragm as depicted by their CT examination.

The patients' ages ranged from 5 to 84 years with a mean age \pm SD $(43.25 \pm 7.57$ years $)$.

\section{Inclusion criteria}

All patients who had a unilateral diaphragmatic disorder that was detected by conventional CT were involved in the study.

\section{Exclusion criteria}

Patients who had a pulmonic (pleuro-pulmonary) cause of hemidiaphragmatic dysfunction including those who had a loss of volume (lobar collapse, pneumonectomy), supradiaphragmatic masses or fibrosis, pleural thickening or collections, in addition to those who had bilateral copula affection as detected by CT.

The local ethics committee approved the study, and all participants gave their informed consent before being included.

All patients were subjected to the following:

1) History taking and clinical examination.

2) MSCT was performed by using a 16-section (Siemens) CT scanner; the patients were examined in the supine position. The field of view was adjusted to obtain a complete anatomical imaging of the chest. The rotation time was $1 \backslash 2 \mathrm{~s}$, tube current was 300-350 mAs, and tube voltage was $120 \mathrm{kVs}$.

3) TAUS with an assessment of the diaphragm.

\section{Imaging technique and diagnostic criteria in sonography}

The following probes were used: (1) a high-frequency linear transducer of 7.5-12 $\mathrm{MHz}$ was used for imaging the diaphragm and measuring its thickness. (2) A lowfrequency curvilinear transducer $3.5-5 \mathrm{MHz}$ is used for assessing the diaphragmatic excursion.

Different ultrasound machines in our department were used (according to their availability at the time of scanning) including Toshiba Aplio 500, General Electric Logic P7, General Electric Logic S8, and General Electric Voluson E6.

\section{Technique}

The examination steps were explained in detail for the patients, and the patients were trained enough for the different respiratory maneuvers performance (normal, deep breathing, and the sniffing).

Patient examinations were done by a well-trained radiology resident (who was blinded from the CT results so as to avoid diagnostic bias) in supine and semi-sitting position during spontaneous respiration. A radiology consultant (who was oriented by the CT results) was present during the TAUS examinations, for direct supervision of the quality of the examinations, to correlate the imaging data obtained by the CT and the TAUS examinations, and to help for solving any conflicts.

The transducer was placed in the following sites to obtain different views of the diaphragm (Fig. 1):

1) Intercostal view: was obtained by transducer placement on the ninth intercostal space in the 


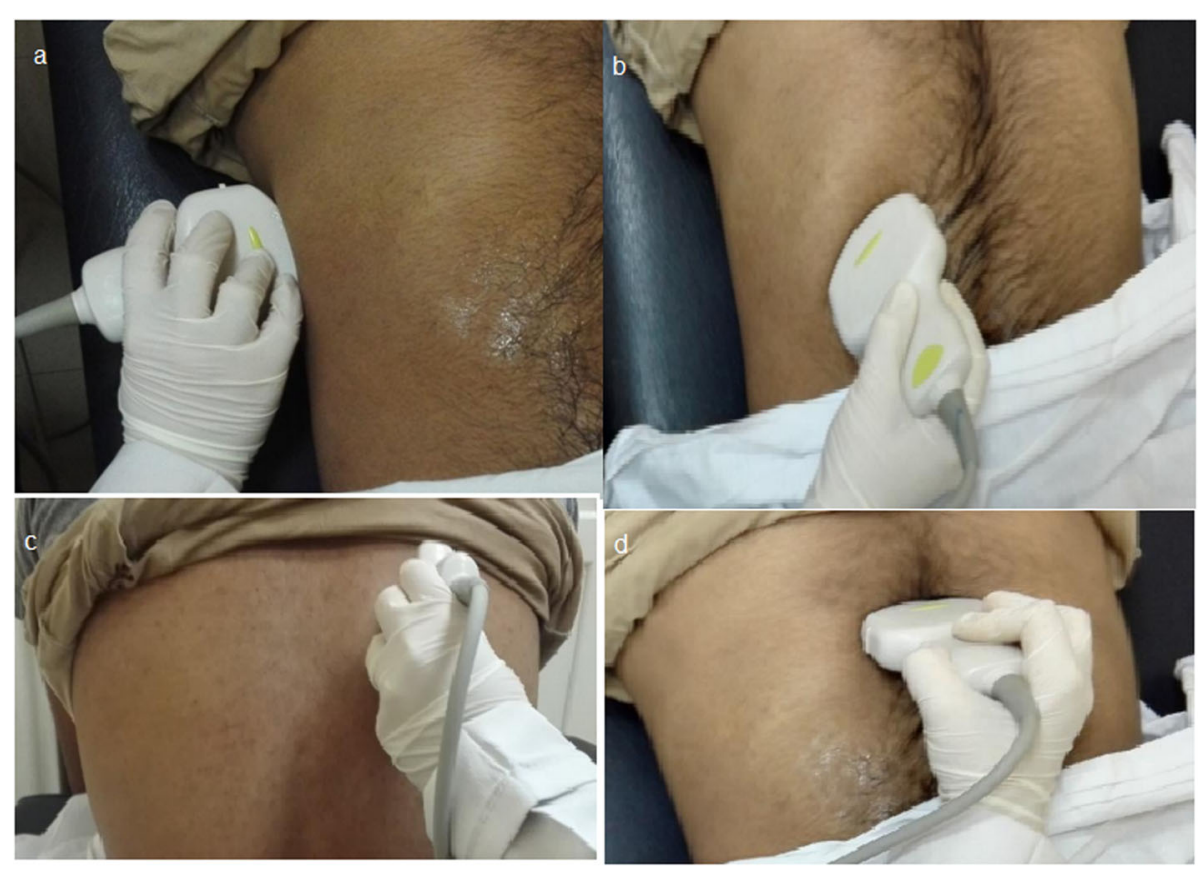

Fig. 1 a-d Photographic pictures demonstrating different probe and patients' positions for diaphragmatic ultrasound evaluation. a Probe positioning into the anterior axillary line to obtain the intercostal view at the zone of apposition. $\mathbf{b}$ Curvilinear probe was placed to obtain the anterior subcostal view. c Patient was sitting when the posterior subcostal view was obtained. $\mathbf{d}$ Subxiphoid view was obtained for qualitative assessment of both hemidaphragms

anterior axillary line. Diaphragm visualization at the zone of apposition using this approach (Fig. 1a).

2) Anterior subcostal view: the transducer was placed between the midclavicular and anterior axillary lines, and then directed medially, cranially, and dorsally (Fig. 1b).

3) Posterior subcostal view: similar to the anterior subcostal view, the transducer was placed in the posterior subcostal region (at the midscapular line); this view required the patients to be seated, which may not be applicable (not done) in all cases (Fig. 1c).

4) Subxiphoid view where the transducer was placed below the xiphoid in a transverse orientation then angulated upward (cranially) for visualization of both sides simultaneously to detect abnormal (paradoxical) motion of one side and to make a side-to-side comparison for excursion parameters (Fig. 1d).

The following measurements were obtained for all patients:

1) Diaphragm thickness using the intercostal approach with a high-frequency linear transducer in B-mode; the thickness was measured at the zone of apposition inferiorly to the costophrenic angle.

2) Thickening fraction that was calculated through Gottesman and McCool formula [8].
(Thickness at end-inspiration-Thickness at endexpiration)/Thickness at end-expiration.

3) Diaphragmatic excursion using the intercostal/ subcostal views with a low-frequency curvilinear transducer in M-mode; the excursion was obtained in normal, deep breathing, and in sniffing also then the amplitude of the interrogated signals was measured, and a comparison between both copulas had been done.

\section{Statistical methods}

Data were coded and entered using the statistical package SPSS (Statistical Package for the Social Sciences) version 25. Data was summarized using frequency (count) and relative frequency (percentage) for categorical data. Standard diagnostic indices including sensitivity, specificity, positive predictive value (PPV), negative predictive value (NPV), and diagnostic efficacy were calculated as described by [9].

\section{Results}

TAUS had been used in this study for evaluation of the dysfunctional hemidiaphragm as reported in the CT examination of the patients included in the present study, where the right hemidiaphragm dysfunction was present in $63.8 \%$ of our patients and the left one dysfunction was present in $36.2 \%$ of patients (Table 1 ). 
Table 1 Demonstrating the type of pathology for each hemidiaphragm and the number of affected patients

\begin{tabular}{lll}
\hline Pathology & Right copula & Left copula \\
\hline 1. Defects/hernia & 2 patients & 5 patients \\
2. Weakness/paralysis & 13 patients & 6 patients \\
$\begin{array}{l}\text { 3. Infra-diaphragmatic } \\
\text { collections(ascites) }\end{array}$ & 8 patients & 2 patients \\
Total & 23 patients & 13 patients \\
& & 36 patients \\
\hline
\end{tabular}

In CT examination, the dysfunctional hemidiaphragm was considered when either a hernia/post-traumatic defects (seen in $19.4 \%$ of our patients, of which $5.5 \%$ were depicted on the right side and $13.8 \%$ were existing on the left side) or elevated copulae (found in $80.6 \%$ of patients, of which $58.8 \%$ were found on the right side and $22.2 \%$ were seen on the left side) (Table 1).

The diaphragmatic defects had been subcategorized to hernias and post-traumatic defects (present in 7 patients $19.4 \%, 5$ on the left side and 2 on the right side) with hernia being seen in 5 cases ( 3 congenital cases and 2 adult cases) and the traumatic defects were present in 2 cases; a left side predominance for hernia and defects was noted in this study (Figs 2, 3, and 4).

A defect/hernia was considered in CT when a discontinuous copula or a gap was present in the affected hemidiaphragm, and the abdominal viscera might be seen protruding through it. In this study, the TAUS showed a high sensitivity, specificity, positive predictive value (PPV), negative predictive value (NPV), and overall accuracy $\{100 \%$ (95\% confidence interval $\mathrm{CI}=59.04$ to $100.00 \%$ ), $100 \%$ ( $95 \% \mathrm{CI}=88.06$ to $100.00 \%$ ), $100 \%, 100 \%, 100 \%$ ( $95 \% \mathrm{CI}=90.26$ to100.00\%), respectively in detection of the defects/hernia as compared to CT (Tables 2 and 3).

An elevated copula was considered in CT when there was a side-to-side difference more than $2 \mathrm{~cm}$ between both copulas and thinning out (atrophy) might be seen; however, this was relevant-by TAUS-to abnormal diaphragmatic thickness (measured by B-mode and the thickening fraction), diaphragmatic weakness or paralysis (measured by M-mode in normal and deep breathing as well as in sniffing) or if there were subphrenic collections or ascites underneath the affected copula. In this study, the TAUS showed a high sensitivity, specificity, PPV, NPV, and overall accuracy $\{96.55 \%$ (95\% CI $=$ 82.24 to $99.9 \%), 100 \%(95 \% \mathrm{CI}=59.04$ to $100.00 \%)$, $100 \%, 87.5 \%(95 \% \mathrm{CI}=50.50$ to $97.96 \%), 97.22 \%(95 \%$ $\mathrm{CI}=85.47$ to $99.93 \%$ ), respectively\} in the detection of these findings as compared to CT (Tables 4 and 5).

A thinned out diaphragm (atrophy) as measured by TAUS was present in 9 cases ( 8 with paralysis and 1 with weakness); the mean thickness for cases with thinned out diaphragm was $1.8 \mathrm{~mm}$.

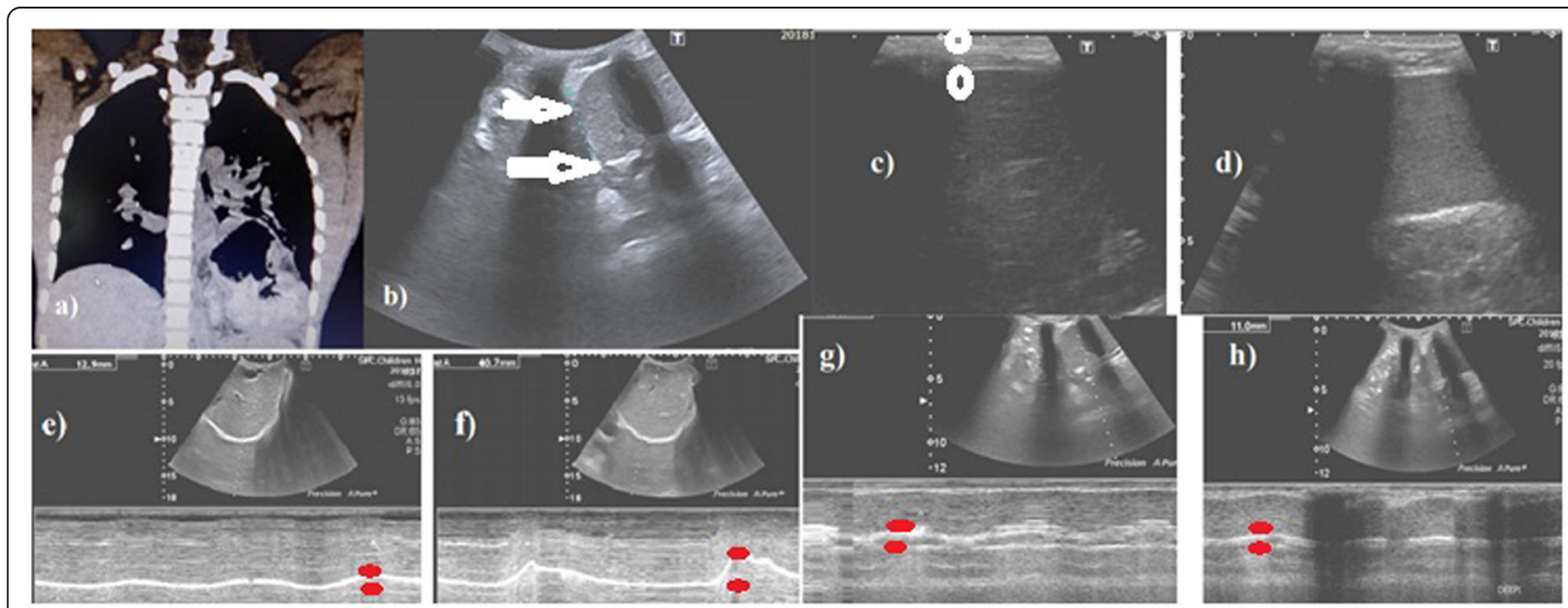

Fig. 2 a-h CT and US images for an 8-year-old male patient presented with a congenital diaphragmatic hernia for preoperative assessment. a Coronal reformatted CT of chest (mediastinal window) showing a large postero-lateral defect in the left hemidiaphragm through which a herniated bowel loops were seen in the left hemi thorax. b An US image-intercostal view_showing the defect that measure $6 \mathrm{~cm}$ (between the side arrows) as in CT and the echogenic bowel loops in the left hemithorax. $\mathbf{c}$, $\mathbf{d}$ US images using the superficial probe through an intercostal view showing an average diaphragmatic thickness (RT $=2.4 \mathrm{~mm}$, between the white circles $/ \mathrm{LT}=2.5 \mathrm{~mm}$ between the white dots) with an adequate thickening fraction (RT $=43 \% / L T=48 \%$ ). $\mathbf{e}-\mathbf{h}$ US images (for functional assessment of the diaphragm) -in intercostal views-showing within normal right diaphragmatic excursion in normal, deep breathing, and sniff test $(=1.2 \mathrm{~cm}$ in normal breathing (e, between the red dots)/= $3.4 \mathrm{~cm}$ in deep breathing between the red dots $/=4 \mathrm{~cm}$ in sniffing (f, between the red dots) and below normal left diaphragmatic excursion in deep breathing and sniff test $(=0.9 \mathrm{~cm}$ in normal breathing $(\mathbf{g}$, between the red dots) $/=1.1 \mathrm{~cm}$ in deep breathing $(\mathbf{h}$, between the red dots $) /=$ $1.3 \mathrm{~cm}$ in sniffing between the red dots); these findings were relevant to a left hemidiaphragmatic weakness with a bowel containing left hemidiaphragmatic hernia 


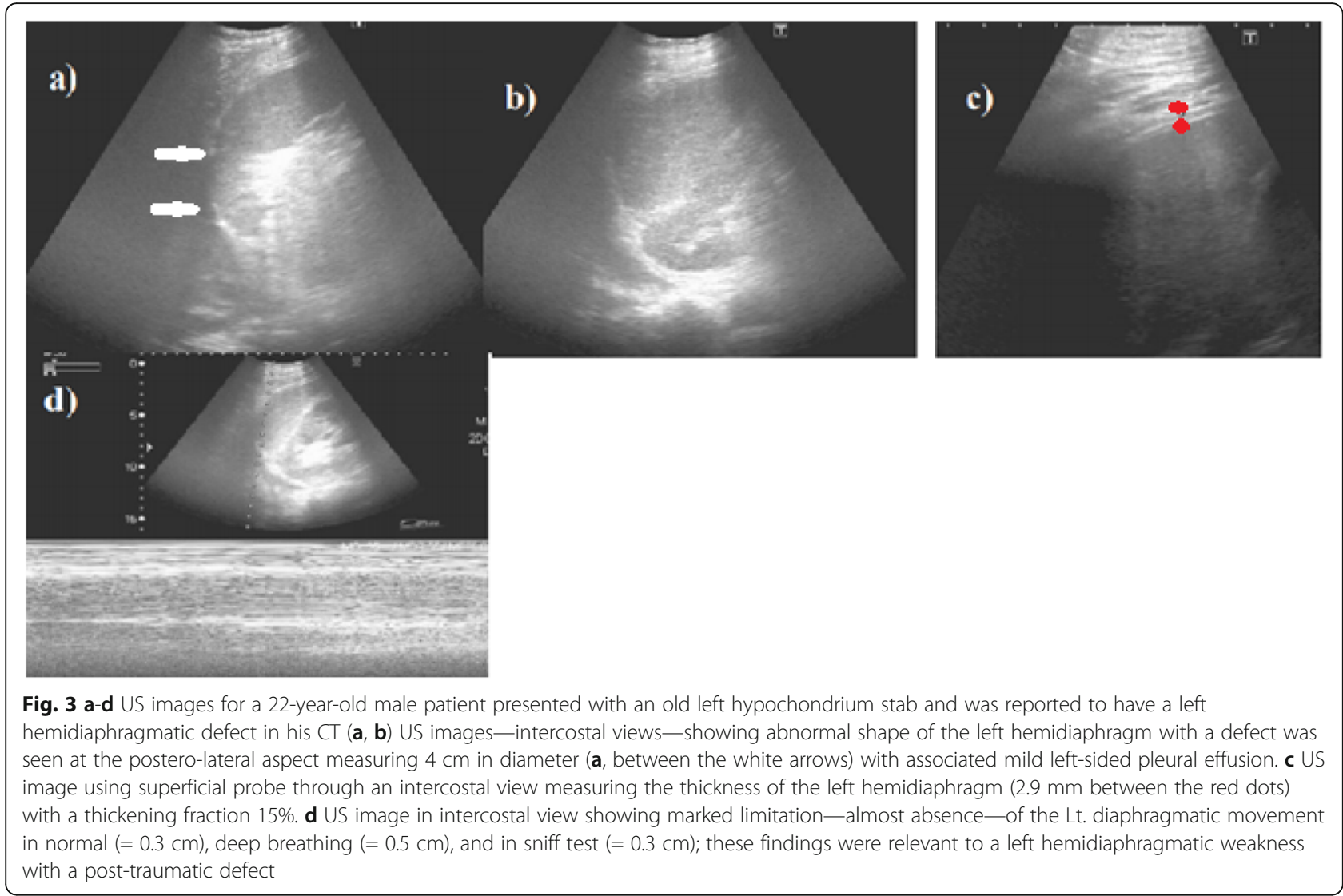

Patients with diaphragmatic weakness/paralysis were present in 19 patients (13 on the right side and 6 on the left one), where a diaphragmatic excursion lower than normal range was present in cases with diaphragmatic weakness $(<0.9 \mathrm{~mm}$ in normal breathing, $<2 \mathrm{~mm}$ in deep breathing, and $<$ $1.4 \mathrm{~mm}$ in sniffing), whereas absent excursion was the salient finding in cases of diaphragmatic paralysis with or without a thickening fraction less than $22 \%$ (Figs. 5 and 6).
Diaphragmatic eventration is defined as a congenital weakness of a segment of the diaphragm, and this was seen in one of our patients (Fig. 6) where the affected hemidiaphragm presented weakness by motion criteria but the copula appeared to be subtotally weakened (not focally weakened by definition); on reviewing the patient history, there was a long-standing constipation, thus chronically elevated abdominal pressure could be the precipitating factor for this-larger than expectedeventration.

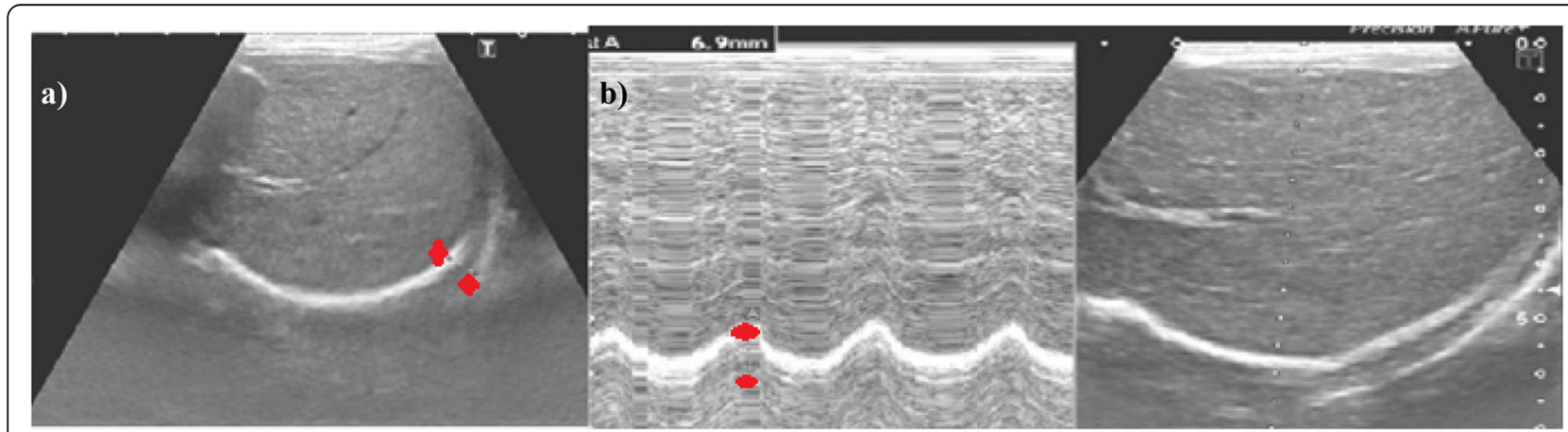

Fig. 4 a, b US images for a 5-year-old male patient presented with a post-open heart surgery dyspnea and an elevated right copula in his CT. a Abnormal shape of the right hemidiaphragm with an overlapping defect was seen measuring $6 \mathrm{~mm}$ in diameter (between the red dots). $\mathbf{b}$ US image in intercostal view showing limited movement of the right hemidiaphragm in normal breathing $(=0.6 \mathrm{~cm}$ between the red dots), and its diagnosis was established as a postoperative right hemidiaphragmatic overlapping defect and weakness 
Table 2 Demonstrating the number of patients-out of total patients (36) — with hemidiaphragmatic defects/hernias as detected by TAUS in comparison to CT

\begin{tabular}{|c|c|c|c|c|c|}
\hline & & $C T$ (def & s (herni & & \\
\hline & & Yes & & No & \\
\hline & & Count & $\%$ & Count & $\%$ \\
\hline US (defects (hernia)) & Yes & 7 & 100.0 & 0 & 0.0 \\
\hline & No & 0 & 0.0 & 29 & 100.0 \\
\hline
\end{tabular}

Patients with elevated diaphragmatic copula due infradiaphragmatic collections were present in 10 cases (8 patients on the right side and 2 on the left one) where the underlying subphrenic collection is evident by the TAUS and with motion criteria like those of diaphragmatic weakness.

\section{Discussion}

Being the most important respiratory muscle, thus the diaphragm had gained special concern for its anatomical and functional disorders by both physical examination and imaging workup [1].

Despite that they might be asymptomatic, the unilateral diaphragmatic disorders had been described as more common than the usually symptomatic bilateral affection [2-4].

In the present study, the accuracy measures of the TAUS had been done in comparison to the CT examinations regarding the diaphragmatic defects and the elevated diaphragmatic copula as reported in the CT.

The diaphragmatic defects, in our study, had included hernias and post-traumatic defects (present in 7 patients $19.4 \%, 5$ on the left side and 2 on the right side) with hernia being seen in 5 cases (3 congenital cases and 2 adult cases), and the traumatic defects were present in two cases: a left side predominance for hernia and defects was noted in this study (Fig. 2, 3, and 4); this had an agreement with the literature, where the congenital posterior hernias are commonly left-sided and more common than the anterior ones [10, 11], and this was explained by the relative protective effect of the liver for the right hemidiaphragm.

Table 3 Demonstrating the accuracy measures of TAUS in comparison to $\mathrm{CT}$ in the detection of the hemidiaphragmatic defects/hernias

\begin{tabular}{lll}
\hline Statistic & Value & $95 \% \mathrm{Cl}$ \\
\hline Sensitivity & $100.00 \%$ & 59.04 to $100.00 \%$ \\
Specificity & $100.00 \%$ & 88.06 to $100.00 \%$ \\
Positive predictive value & $100.00 \%$ & \\
Negative predictive value & $100.00 \%$ & \\
Accuracy & $100.00 \%$ & 90.26 to $100.00 \%$ \\
\hline
\end{tabular}

Table 4 Demonstrating the number of patients - out of total patients (36) — with hemidiaphragmatic weakness/paralysis as detected by TAUS relevant to an elevated diaphragmatic copula in $\mathrm{CT}$

\begin{tabular}{|c|c|c|c|c|c|}
\hline & & \multicolumn{4}{|c|}{ CT (elevated copula) } \\
\hline & & \multicolumn{2}{|l|}{ Yes } & \multicolumn{2}{|l|}{ No } \\
\hline & & Count & $\%$ & Count & $\%$ \\
\hline \multirow{2}{*}{$\begin{array}{l}\text { US (abnormal thickness- } \\
\text { weakness-paralysis) }\end{array}$} & Yes & 28 & 96.6 & 0 & 0.0 \\
\hline & No & 1 & 3.4 & 7 & 100.0 \\
\hline
\end{tabular}

Post-traumatic defects can occur either by blunt or penetrating injuries; the blunt injuries are usually under diagnosed at the initial presentation probably due to overshadowing by other associated injuries. CT was considered as the reference standard for both types of injury, and many CT signs had been described as clues for the diagnosis by Desir and Ghaye; they also had reported that the left hemidiaphragm was more liable to injury than the right one and explained this by a multifactorial issues, including the liver support to the right hemi diaphragm. Additionally, it had an inherent resistance greater than the left one. Moreover, the congenital embryonic weakness of the postero-lateral aspect of the left hemidiaphragm might further weaken the left one [12]. We had a great match with such data and thus we used the CT as the reference standard in hemidiaphragmatic defects and hernias; however, in our study, TAUS showed a great sensitivity and specificity $\{100 \%$ (95\% CI $=59.04$ to $100.00 \%)$ and $100 \%$ (95\% CI $=88.06$ to $100.00 \%$ ), respectively $\}$ in this regard as compared to CT (the reference standard), but actually the number of cases in the present study was considerably lower and thus considered as a limitation despite the high accuracy measures and the matched data of the lesion location.

Although there is no consensus on the TAUS sensitivity and specificity in diagnosis of diaphragmatic rupture, there had been many reports in the literature that aided in the diagnosis including direct visualization of the diaphragmatic defects (this was also seen in our cases Fig. 3 and 4), visualization of some abdominal viscera above the diaphragm, and by

Table 5 Demonstrating the accuracy measures of hemidiaphragmatic abnormal thickness, weakness/paralysis as detected by TAUS relevant to an elevated hemidiaphragmatic copula in CT

\begin{tabular}{lll}
\hline Statistic & Value & $95 \% \mathrm{Cl}$ \\
\hline Sensitivity & $96.55 \%$ & 82.24 to $99.91 \%$ \\
Specificity & $100.00 \%$ & 59.04 to $100.00 \%$ \\
Positive predictive value & $100.00 \%$ & \\
Negative predictive value & $87.50 \%$ & 50.50 to $97.96 \%$ \\
Accuracy & $97.22 \%$ & 85.47 to $99.93 \%$ \\
\hline
\end{tabular}




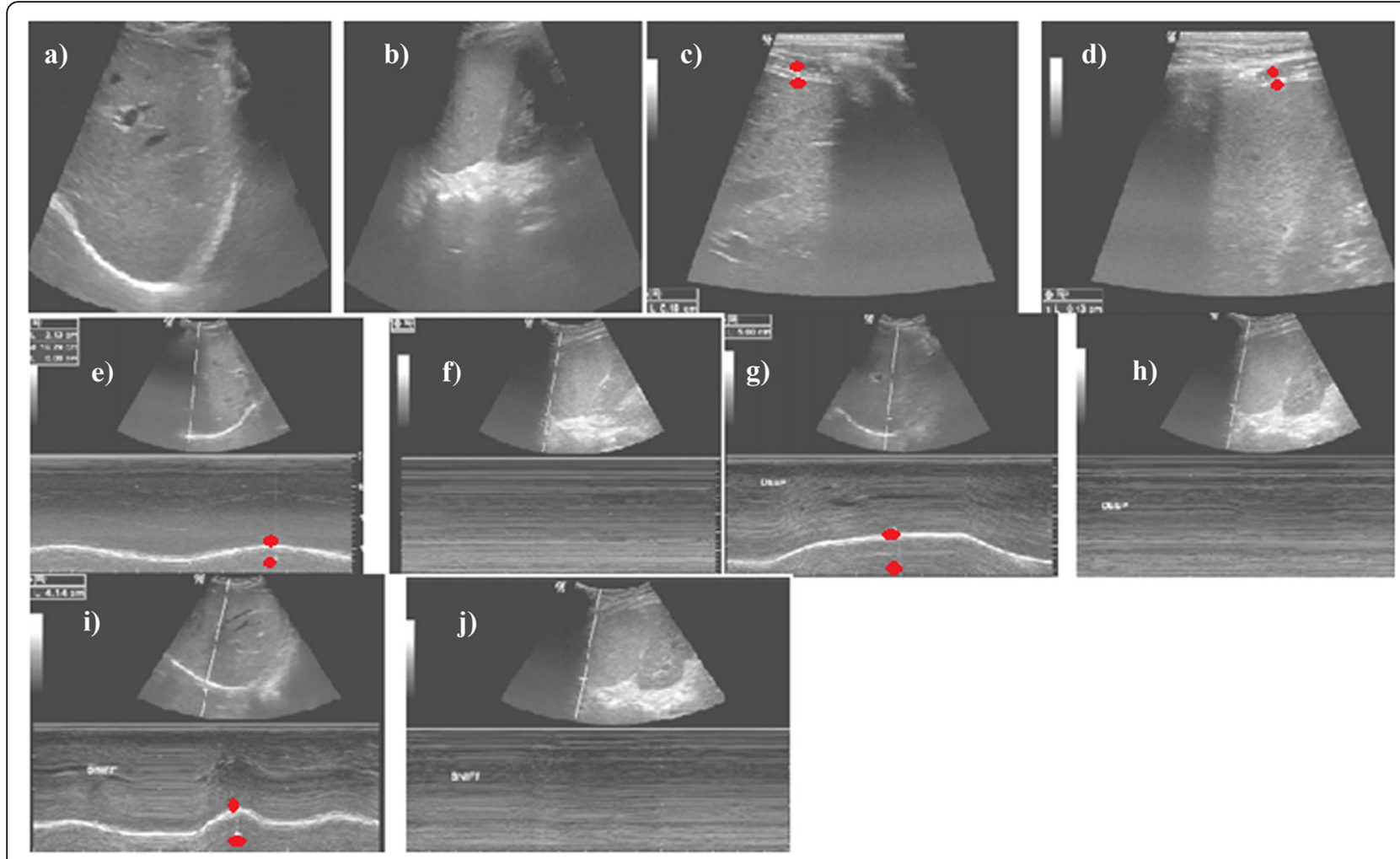

Fig. 5 a-j US images for a 52-year-old male patient with a history of cardiothoracic surgery 6 months ago presented with elevated left copula of the diaphragm in his CT. a, b US images-anterior subcostal view (a) and intercostals view (b) showing normal US appearance of both hemidiaphragms with no defect detected on either side. c, d US image using superficial probe through an intercostal view showing a diaphragmatic thickness (RT $=1.9 \mathrm{~mm}$ ( $\mathbf{c}$, between the red dots) $/ \mathrm{LT}=1.3 \mathrm{~mm}$ \{thin, atrophic $\}$ (d, between the red dots)) with a thickening fraction $(\mathrm{RT}=45 \% / \mathrm{LT}=10 \%)$. e-j Anterior subcostal and intercostal views used for assessment of diaphragmatic excursion (RT $=2.1 \mathrm{~cm}$ in normal breathing (e, between red dots) $/=5 \mathrm{~cm}$ in deep breathing $(\mathbf{g}$, between the red dots) $/=4 \mathrm{~cm}$ in sniffing (i, between the red dots) and the left side showed absent movement in normal (f), deep breathing (h), and sniffing (j). Findings were consistent with left hemidiaphragmatic paralysis to be likely a postoperative sequlae

M-mode; abnormal diaphragmatic excursion was readily seen [13], and this also had been seen in our study (Figs 2, 3, and 4).

In our limited experience, TAUS could be considered as a beneficial initial diagnostic tool for diaphragmatic injury especially if the motion assessment by M-mode was added to the anatomical scan; this was also supported by Bothwell et al. who documented the additional advantage of the dynamic assessment of the diaphragm over the plain radiography in this concern [13].

An elevated diaphragmatic copula in CT is considered as a sign of diaphragmatic dysfunction and could be a diaphragmatic weakness, paralysis, or due to subdiaphragmatic collection, and this was relevant-by TAUS-to abnormal diaphragmatic thickness (measured by B-mode and the thickening fraction), diaphragmatic weakness or paralysis (measured by M-mode in quiet and deep respiration as well as in sniffing), or if there were subphrenic collections or ascites underneath the affected copula.

Goligher and his co-workers stated that diaphragm thickness depends mainly on the muscle mass; thus, a reduced diaphragm thickness might indicate its atrophy. Measurement of the diaphragm thickness is feasible by using TAUS, where the hepatic window can facilitate the measurement of the right hemidiaphragm than the less accessible left one. They had estimated the normal diaphragm thickness in ventilated patients $(2.4 \pm 0.8 \mathrm{~mm})$, and diaphragmatic atrophy had been identified as values below $2 \mathrm{~mm}$ [14]. A rather similar study by McCool et al., stated that the average thickness of the diaphragm is $2.2-2.8 \mathrm{~mm}$ in healthy volunteers and $1.3-1.9 \mathrm{~mm}$ in a paralyzed diaphragm. They also had reported the same issue as a diaphragm thickness less than $2 \mathrm{~mm}$ had been suggested as a cut-off value to define diaphragmatic atrophy [15]. A thinned out diaphragm as measured by TAUS was present in 9 of our cases (8 with paralysis and 1 with weakness out of 19 cases with paralysis and weakness). The mean thickness for cases with thinned out diaphragm was $1.8 \mathrm{~mm}$; however, missing 10 cases out of 19 cases were a considerable issue. Kantarci et al. had explained this as the thickness could vary according to the site of measurement and the phase of the 


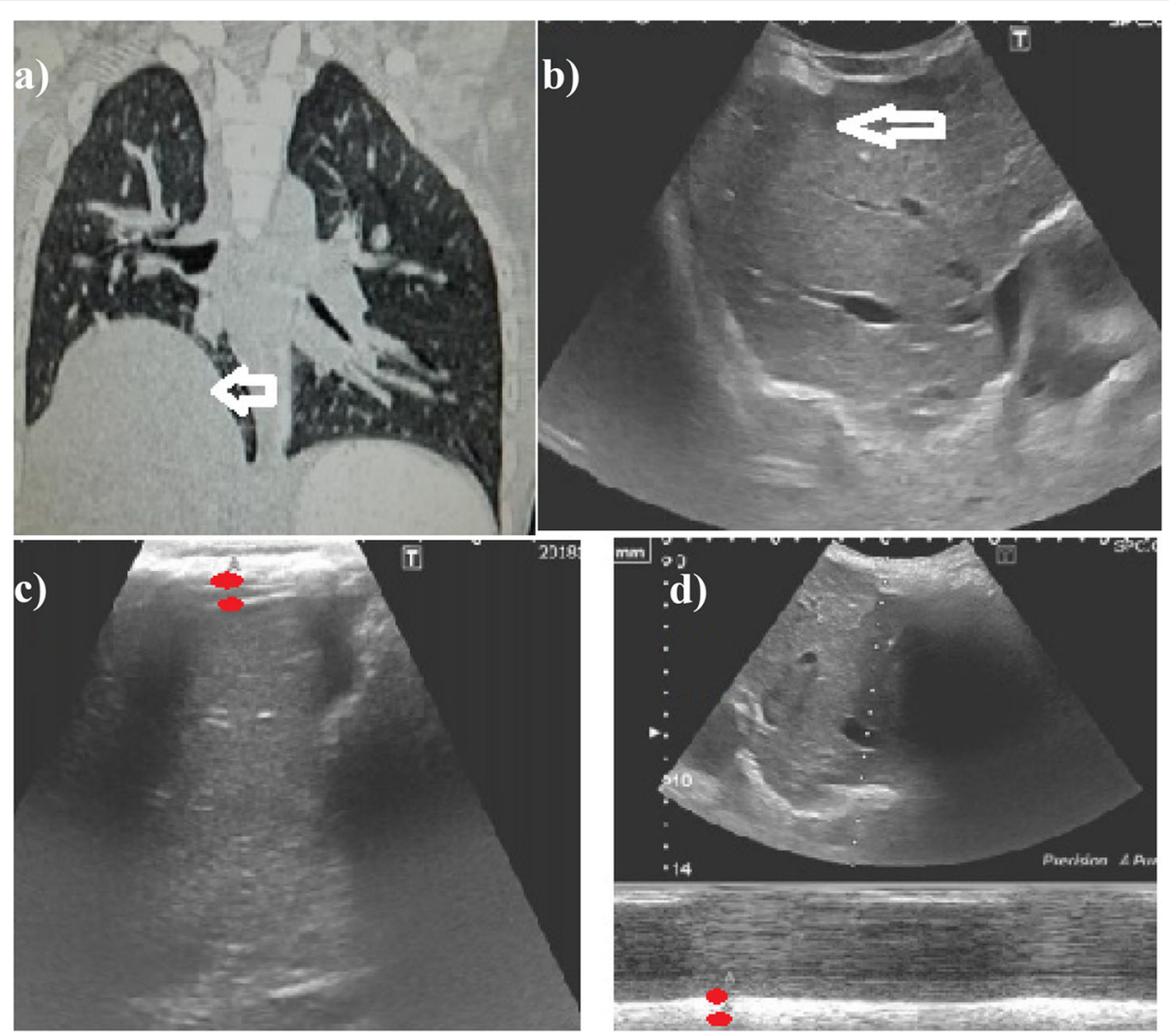

Fig. $\mathbf{6}$ a-d CT and US images for a 6-year-old female patient presented with elevated right copula of the diaphragm that was discovered accidentally on chest CT. a Coronal formatted CT of chest (lung window) showing an elevated right diaphragmatic copula (open white arrow) with no mediastinal shift. b An US image-intercostal view_showing abnormal focal bulge of the anteromedial aspect of the right hemidiaphragm (open white arrow) with no defect detected. c US image using superficial probe through an intercostal view showing abnormal focal thinning out of the right hemidiaphragm ( $1.7 \mathrm{~mm}$ between the red dots) with a thickening fraction $22 \%$. $\mathbf{d}$ US image in intercostal view showing limited diaphragmatic movements in normal breathing $=0.8 \mathrm{~cm}$, deep breathing $=1.8 \mathrm{~cm}$, and in sniff test $=1 \mathrm{~cm}$ between the red dots in $\mathbf{d}$; these findings were relevant to a right hemidiaphragmatic eventration (a dromedary hump) with weakness

respiratory cycle (start of inspiration or end of expiration), thereby, a thickness as the sole parameter could miss a recently paralyzed diaphragm that could attain a normal thickness for a while, and on the other hand, it could identify an atrophic one in individuals with low body weight [16], this was also matching our study, where some patients with diaphragmatic weakness ( not yet paralyzed) that might preserve some muscle mass and action could have a thickness a little bit more than the lower limit value of $1.9 \mathrm{~mm}$. Therefore, there is an agreement between our study and many literatures that the degree of diaphragm thickening (thickening fraction) had been assumed to be a more sensitive method than the measurement of thickness alone $[4,8,16,17]$.

As the muscles generally thicken during contraction due to fiber shortening, thus, the increase in the diaphragmatic thickness during contraction (inspiration) could be used as an indirect indicator of the muscle fiber contraction, and this was described and measured by a general formula of Gottesman and McCool, a thickening fraction $=$ (thickness at end-inspiration-thickness at end-expiration)/thickness at end-expiration [8], and it had reported in the literature to have a range from 22 to $78 \%$ with a mean thickening fraction $=38.4 \%[8,18-21]$. They concluded that diaphragmatic thickness and the thickening fraction could detect diaphragmatic paralysis and confirm diaphragmatic atrophy in long-standing paralysis with poor thickening on inspiration; Jung et al. had documented that a thickness variation of $<20 \%$ was a predictor for failure of weaning from mechanical ventilation due to diaphragmatic dysfunction [22]. In our study, the results had a concordance with the previous studies where the thickness and the thickening fraction were utilized as parameters for evaluation of the diaphragmatic dysfunction not only for assessment of the muscle mass but also emphasizing the value of the dynamic evaluation of the diaphragmatic contraction through assessment of the thickening fraction and actually, this was done even in cases with diaphragmatic defects and hernias as this-from our point of view-could 
give an idea about the restoration of the diaphragmatic function after surgical repair if it would be done; this is eventually considered as merit of the TAUS other than measuring the size of the defect.

It should be mentioned that TAUS had the advantage of an indirect assessment of the degree of diaphragmatic contraction (through the measurement of the thickening fraction) over other imaging modalities that can barely assess the diaphragmatic motion and its paradox.

Another role for the TAUS could be added in diaphragmatic function evaluation which was the diaphragmatic motion by M-mode and assessment of the degree of excursion where the diaphragm was assessed in quiet and deep breathing as well as with sniffing.

The diaphragmatic excursion should be done using the curvilinear probe, in M-mode, and measured from the point of the maximal excursion to the baseline, in quiet breathing and in the sniffing; in deep breathing, the measurement should be from the highest to the lowest point of excursion [23], and this was done in the present study as well.

Boussuges et al. had demonstrated that M-mode ultrasonography was a reproducible method for assessing hemidiaphragmatic movement [24].

Diaphragm weakness was indicated by decreased (lessthan-normal) amplitudes of excursion on deep breathing with or without paradoxical motion upon sniffing, whereas a diaphragmatic paralysis was indicated by diminished (absence of) excursion with quiet and deep breathing and with absence of motion or paradoxical movement on sniffing (Figs. 5 and 6). The direction of diaphragmatic motion could be identified by the TAUS and correlated to the respiratory phases and paradoxical motion was identified when the diaphragm was moving away from the probe in the inspiratory phase (the reverse of the expected [24-26].

In our study, diaphragmatic weakness was present in 9 cases that was presented in CT with an elevated copula, and the TAUS revealed abnormal thickening fraction, in addition to the decreased excursion amplitudes; hereby, we had met an important term, which was the diaphragmatic eventration that was defined in the literature by a congenital focal weakness of the diaphragm and commonly occur in the anteromedial aspect of the right copula and radiographically forms a bulge known as hump that can be identified by either plain radiography or CT [23]. It was seen in one of our cases (Fig. 6), and a differentiation from hernia should be done where the eventration has a continuous but focally weak segment while hernia and defects had a usually discontinuity through protrusion of any abdominal viscera.

By definition, eventration should occur in a congenital weak focal segment in otherwise normal diaphragm [23], which was not the case in our patient where the entire diaphragm was weakened (Fig. 6). Our explanation was that, in reviewing the literature, it had been showed that
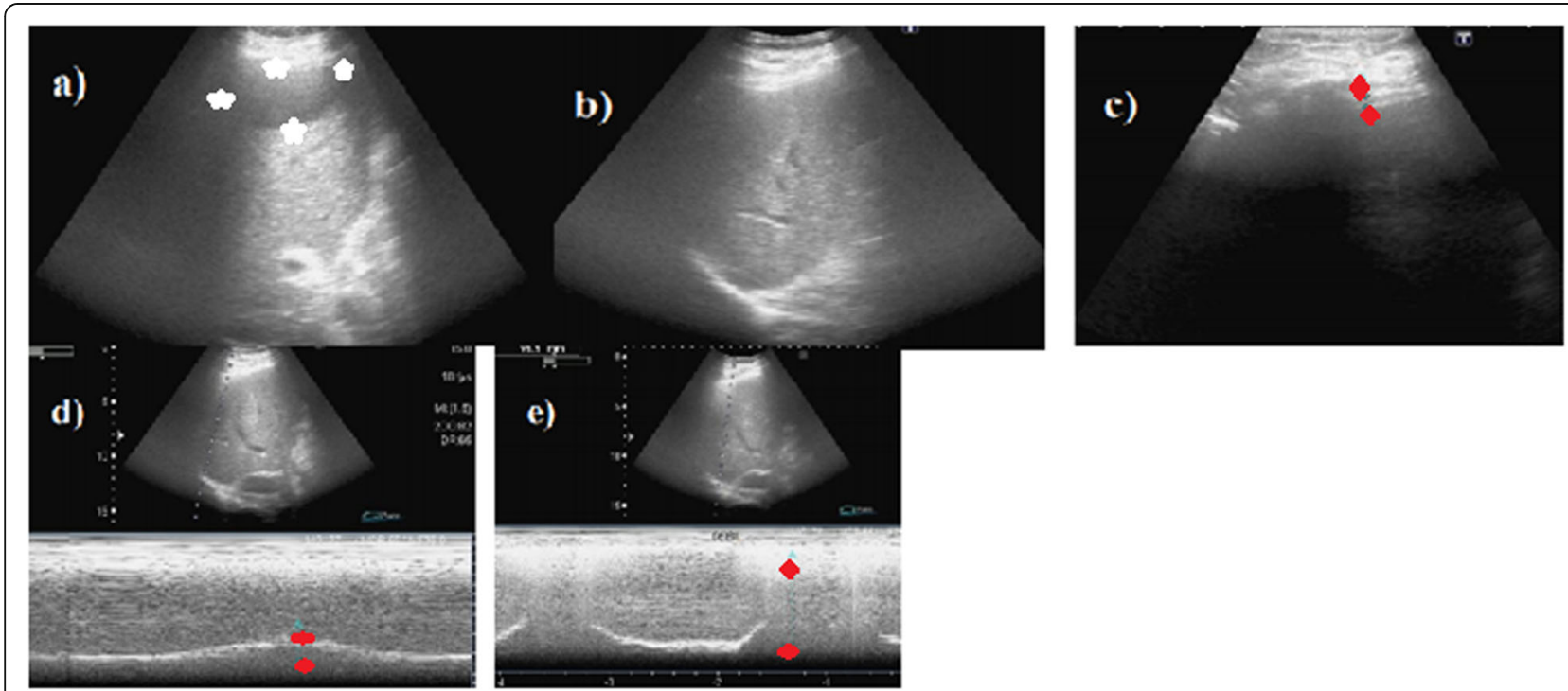

Fig. 7 a-e US images for a 72-year-old male patient presented with fever and abdominal pain, and his CT examination showed a right copula elevation. a, b US images_-intercostals views_-showing a loculated subphrenic collection with internal echoes (complex collection), and no internal vascularity was detected; it is seen measuring $4 \times 5 \mathrm{~cm}$ (a, between the white asterisks) with normal US appearance of the right hemidiaphragm showing no defects (b). c US image using superficial probe through an intercostal view showing a mildly thickened right hemidiaphragm $=3.7 \mathrm{~mm}$ (between the red dots) with adequate thickening fraction (= 48\%) $\mathbf{d}$, e Intercostal views used for the assessment of diaphragmatic excursion ( $(\mathrm{RT}=3 \mathrm{~cm}$ in normal breathing $(\mathbf{d}$, between the red dots) $/=9.3 \mathrm{~cm}$ in deep breathing $(\mathbf{e}$, between the red dots)/=7.8 $\mathrm{cm}$ in sniffing) showing a picture of elevated right diaphragmatic copula with thickening secondary to a right subphrenic collection likely an abscess 
the eventration increases with elevated abdominal pressure and could actually widen and increase with time and may involve the entire hemidiaphragm by conditions related to an increased abdominal pressure, like obesity, ascites, repeated childbirth, and in constipated individuals and heavy object lifters [23], and our patient had a relevant history that had supported this explanation.

In concordance with Boon et al. and Chavhan et al., our study had shown a high sensitivity and specificity $(96.55 \%$ and $100 \%$ sensitivity and specificity, respectively, with an overall accuracy $97.2 \%$ compared to $93 \%$ and $100 \%$ sensitivity and specificity, respectively, in theirs) in evaluation of a hemidiaphragmatic dysfunction either due to neuromuscular causes (paralysis or weakness) or through the visualization of the infradiaphragmatic causes (ascites or collections) (Fig. 7). They considered the TAUS was superior to fluoroscopy in functional evaluation of the diaphragm [27, 28]; in our study, 10 cases were identified to have an infradiaphragmatic collections, where the TAUS had an excellent depiction of the subphrenic collections and could readily give an idea about its nature as a simple, septated, or complex particulate collections, like those in subphrenic abscesses (Fig. 7).

It should be mentioned that the measurement of excursion depends on maximal inspiratory and voluntary action of the patient and this should be considered for the interpretation of the cut-off values [25]; however, it had been reported that the normal side-to-side difference of excursion between both diaphragmatic copulas should be less than $50 \%$ [29]; a right-to-left ratio of excursion had been documented to be $0.5-2.5$ in normal breathing and 0.5-1.6 in deep breathing [24]. We had obtained benefit at this point, as it had utilized the patient's normal side as a reference standard for the affected copula, but this benefit had been lacking in cases with bilateral affection of the diaphragmatic copulas (those who were excluded from our study).

Ultrasound had been proved as a clinically valuable diagnostic modality, but it did have some limitations. The first one was being an examiner-dependent technique, and the second one was a relatively low number of cases presented by hernia and defects. In our study, which might be taken in consideration, in spite of the high obtained accuracy measures, thus, further research with a larger sample volume for hernia and defects should be proposed; the last one was that the measurement of the excursion depends on the maximum voluntary inspiration done by the patients, and this consequently had limited the interpretative data and the usage of general cut-off values of the amplitudes of excursion in a non-homogenous population sample; however, this factor was related to the patient compliance and general condition; thus, a conscious and cooperative patient can help to obtain a maximal inspiratory effort, and the left-right side comparison had provided good control in our study where only one side was a dysfunctional.

\section{Conclusion}

Trans-abdominal ultrasound (TAUS) could accurately assess the diaphragmatic thickness and provides a realtime image of a dysfunctional hemidiaphragm due to a diaphragmatic and infradiaphragmatic causes as well as the diaphragmatic defects with high sensitivity and specificity in comparison to $\mathrm{CT}$.

\section{Abbreviations \\ TAUS: Trans-abdominal ultrasound; CT: Computed tomography; MRI: Magnetic resonance imaging}

\section{Acknowledgements \\ Not applicable.}

\section{Authors' contributions}

$A A B$ is the corresponding author and had supervised the ultrasound examinations and the interpretation of the corresponding correlative image studies if available in the research work. AAM had the main share in editing of the manuscript and reference collection. SAM had the initial clinical assessment of the patients in the study and in the revision of the manuscript. YYS is the head of our team who had the idea of the research and had supervised the patient selection and in the evaluation of the CT findings and final revision of the manuscript. All authors had revised and approved the final submitted form of the manuscript.

\section{Funding}

The author had no fund for this research and had no competing interests.

\section{Availability of data and materials}

All data are available on a software system owned by each of the authors and the corresponding author has the authority to respond if there is any query.

\section{Ethics approval and consent to participate}

The protocol was reviewed and approved by the local ethics committee of the Radiology Department, Cairo University.

The reference number is not applicable.

All patients had given their written consents for participation in this work.

\section{Consent for publication}

All patients had given their written consents for publication.

Competing interests

All authors had no competing interests.

\section{Author details}

${ }^{1}$ Radiology Department, Faculty of Medicine, Cairo University, Cairo, Egypt. ${ }^{2}$ Chest Diseases Department, Faculty of Medicine, Cairo University, Cairo, Egypt.

Received: 27 November 2019 Accepted: 16 July 2020 Published online: 07 August 2020

\section{References}

1. Ünal Ö, Arslan H, Uzun K et al (2000) Evaluation of diaphragmatic movement with MR fluoroscopy in chronic obstructive pulmonary disease. Clinical imaging 24(6):347-350

2. Dubé BP, Dres M (2016) Diaphragm dysfunction: diagnostic approaches and management strategies. Journal of clinical medicine 5(12):113

3. Qureshi, A. (2009). Diaphragm paralysis. In Seminars in respiratory and critical care medicine (Vol. 30, No. 03, pp. 315-32).

4. Nason LK, Walker CM, McNeeley MF et al (2012) Imaging of the diaphragm: anatomy and function. Radiographics 32(2):E51-E70 
5. Ricoy, J., Rodríguez-Núñez, N., Álvarez-Dobaño, J. M., et al. (2018). Diaphragmatic dysfunction. Pulmonology.

6. Boon AJ, Harper CJ, Ghahfarokhi LS et al (2013) Two-dimensional ultrasound imaging of the diaphragm: Quantitative values in normal subjects. Muscle \& nerve 47(6):884-889

7. Ghigna MR, Crutu A, Florea V et al (2016) The role of endobronchial ultrasound-guided fine needle aspiration in the diagnosis of pleural mesothelioma. Cytopathology 27(4):284-288

8. Gottesman E, McCool F (1997) Ultrasound evaluation of the paralyzed diaphragm. American Journal of Respiratory and Critical Care Medicine 155(5):1570-1574

9. Galen RS (1980) Predictive values and efficiency of laboratory testing. Pediat J Clin North Am 27:861-869

10. Deprest J, Brady P, Nicolaides K et al (2014) Prenatal management of the fetus with isolated congenital diaphragmatic hernia in the era of the TOTAL trial. Seminars in Fetal and Neonatal Medicine 19(6):338-348

11. Mullins ME, Stein J, Saini SS et al (2001) Prevalence of incidental Bochdalek's hernia in a large adult population. AJR Am J Roentgenol (full text) Pubmed citation. AJR Am J Roentgenol. 177(2):363-366

12. Desir A, Ghaye B (2012) CT of Blunt Diaphragmatic Rupture. RadioGraphics 32(2):477-498

13. Bothwell J, Della-Giustina D, Laselle B et al (2011) Crit Ultrasound J 3:153. https://doi.org/10.1007/s13089-011-0079-y

14. Goligher EC, Laghi F, Detsky ME et al (2015) Measuring diaphragm thickness with ultrasound in mechanically ventilated patients: feasibility, reproducibility and validity. Intensive care medicine 41(4):642-649

15. McCool FD, Manzoor K, Minami T (2018) Disorders of the Diaphragm. Clinics in chest medicine 39(2):345-360

16. Kantarci F, Mihmanli I, Demirel MK et al (2004) Normal diaphragmatic motion and the effects of body composition: determination with M-mode sonography. Journal of ultrasound in medicine 23(2):255-260

17. Summerhill EM, El-Sameed YA, Glidden TJ et al (2008) Monitoring recovery from diaphragm paralysis with ultrasound. Chest 133(3):737-743

18. Cohn D, Benditt JO, Eveloff S et al (1997) Diaphragm thickening during inspiration. J Appl Physiol. 83(1):291-296

19. Boon AJ, Harper CJ, Ghahfarokhi LS, et al (2013). Two-dimensional ultrasound imaging of the diaphragm: Quantitative values in normal subjects. Muscle Nerve. 29:47(6):884-889.

20. Grosu HB, Lee YI, Lee J et al (2012) Diaphragm muscle thinning in patients who are mechanically ventilated. Chest 142(6):1455-1460

21. Zambon M, Beccaria P, Matsuno J et al (2016) Mechanical ventilation and diaphragmatic atrophy in critically ill patients. Critical Care Medicine. 44(7): 1347-1352

22. Jung B, Moury PH, Mahul M et al (2016) Diaphragmatic dysfunction in patients with ICU-acquired weakness and its impact on extubation failure. Intensive care medicine 42(5):853-861

23. Matamis D, Soilemezi E, Tsagourias M et al (2013) Sonographic evaluation of the diaphragm in critically ill patients. Technique and clinical applications. Intensive care medicine 39(5):801-810

24. Boussuges A, Gole Y, Blanc P (2009) Diaphragmatic motion studied by mmode ultrasonography: methods, reproducibility, and normal values. Chest 135(2):391-400

25. Lerolle N, Guérot E, Dimassi S et al (2009) Ultrasonographic diagnostic criterion for severe diaphragmatic dysfunction after cardiac surgery. Chest 135(2):401-407

26. Epelman M, Navarro OM, Daneman A et al (2005) M-mode sonography of diaphragmatic motion: description of technique and experience in 278 pediatric patients. Pediatric radiology 35(7):661-667

27. Boon AJ, Sekiguchi $H$, Harper CJ et al (2014) Sensitivity and specificity of diagnostic ultrasound in the diagnosis of phrenic neuropathy. Neurology 83(14):1264-1270

28. Chavhan GB, Babyn PS, Cohen RA et al (2010) Multimodality imaging of the pediatric diaphragm: anatomy and pathologic conditions. Radiographics 30(7):1797-1817

29. Urvoas E, Pariente D, Fausser C et al (1994) Diaphragmatic paralysis in children: diagnosis by TM-mode ultrasound. Pediatric radiology 24(8): 564-568

\section{Publisher's Note}

Springer Nature remains neutral with regard to jurisdictional claims in published maps and institutional affiliations.

\section{Submit your manuscript to a SpringerOpen ${ }^{\circ}$ journal and benefit from:}

- Convenient online submission

- Rigorous peer review

- Open access: articles freely available online

- High visibility within the field

- Retaining the copyright to your article

Submit your next manuscript at $\boldsymbol{\nabla}$ springeropen.com 